\title{
A XBEE based WSN with GSM Technology to Monitor Paddy Field Environment
}

\author{
A. Santhosh \\ M.Tech \\ Vardhaman College of Engineering \\ Kacharam, Hyderabad, India
}

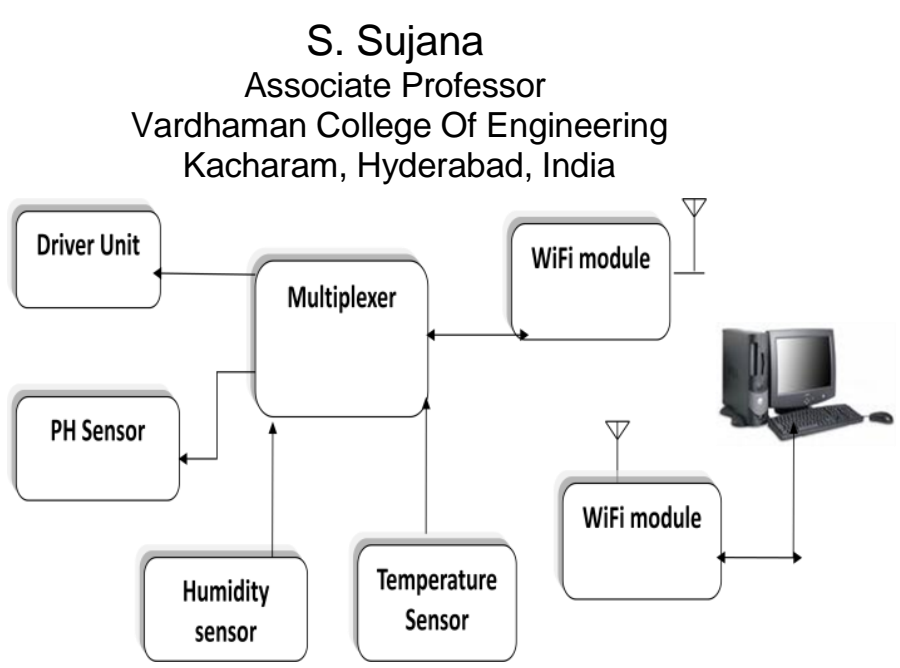

Present system doesn't aware where and when irrigation is needed also it doesn't aware how much fertilization needed such that water and power consumptions are very high.

\section{Proposed system}

Proposed system minimizes water loggings in land. So, it can conserve water and power. By using wireless technology and advanced processors monitoring of various sensors is possible. And farmers can decide amount of fertilization is needed for a particular field by observing recorded data from $\mathrm{pH}$ sensors. Water consumption is minimized by controlling motor (using relay circuit) wirelessly with help of sensed data from water level sensors.

\section{INTRODUCTION}

Sensor networks are developed for variety of applications and awareness of implementing technology into an agricultural environment has increased. Wireless sensor network can reduce human effort and time required for monitoring and controlling a particular field. Various sensors are deployed in critical site without need to put personnel in hazardous and abnormal situations and reduce labour cost. By using technology one can measure factors such as humidity, water level, $\mathrm{pH}$ level, light detection and temperature.

A system is developed, which can monitor environment factors, where data can be sent or received through the xbee wireless medium works at $2.4 \mathrm{GHz}$ band, an operator who is placed at remote location issues commands. Using advanced ARM processor technology many sensors are deployed in the site and GSM technology is used to inform to the personnel if abnormal situation arises. The conceptual diagram is shown below 


\section{Block diagram:}

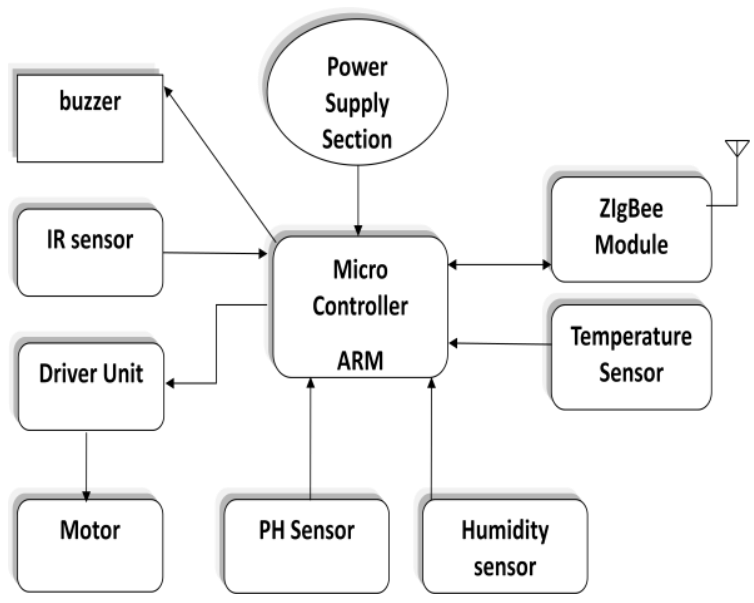

Fig1. Paddy crop field

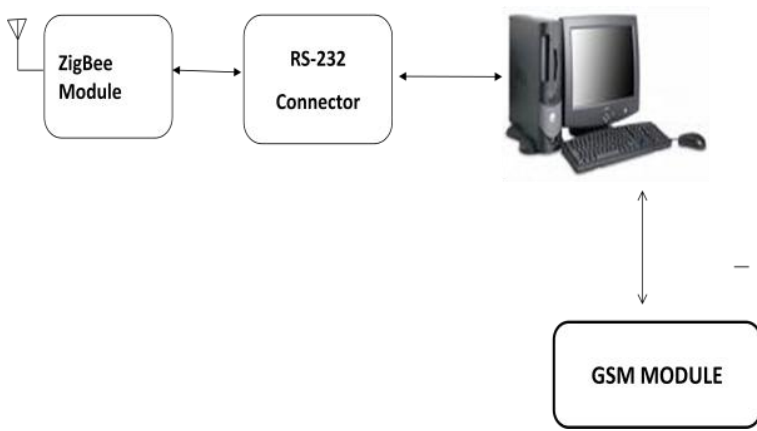

Fig2. Monitoring field

\subsection{Block diagram description}

The proposed system consist two sections one is paddy crop field section and another one is field crop monitoring section. Crop field section consist sensor with ARM processor, temperature sensor is used to find the temperature of atmosphere, Humidity sensor is used to find moisture of soil, ph sensor is used to find the level of the water and IR sensor is used to detect when ever any object occur and those information will send to field crop monitoring section using Zigbee module, it is protocol it is work based request and acknowledge. In monitoring section information will display using Visual basic.

\section{SYSTEM CONFIGURATION}

The system is developed based on xbee module works at 2.4 $\mathrm{GHz}$ band in order to communicate data to the server. The xbee module is connected various sensors which are deployed in the field through the ARM 732 bit microcontroller which has capability to connect many sensors in a site and monitoring and controlling of motor in the site possible such that water consumption and power consumption can be reduced

Detailed descriptions of each module which are deployed in the field are:

\subsection{Zigbee Module}

Zigbee is a wireless module works at $2.4 \mathrm{GHz}$ band meets standard IEEE 802.15 .4 , by using this module as a coordinator a wireless sensor network is constructed and network nodes are sensor nodes which are deployed to monitor agricultural environment and data processing as well as controlling is done by much advanced $\mu \mathrm{C}$, is programmed using "embedded C".

In zigbee network there is only one coordinator per network, number of end points and routers are depend on the application requirement. Before joining a Zigbee network a device with an IEEE 802.15.4 radio has a 64 bit address (this is a global unique number) when a device joins a network it receives 16 bit address called the net work address. It is to establish communication between the devices. After device has joined to the Zigbee network it can send command to other device on the same network. Zigbee has its own library functions like: XBEE_ROLE, NODEID_STR.

The Xbee/Xbee-PRO radio frequency Modules are designed to operate within the ZigBee protocol and support the unique needs of low-power, low cost, reliable wireless sensor networks. The module needs minimal power and provides more reliable delivery of data among remote devices. The module operates in the ISM $2.4 \mathrm{GHz}$ frequency band and is compatible with the following:

XBee USB Adapter ,XBee RS-232 Adapter, XBee Wall Router, XBee RS-485 Adapter, XBee RS-232 PH (Power Harvester) Adapter, XBee Sensor Adapter, XBee Analog I/O Adapter, XBee Digital I/O Adapter, Connect Port X Gateways

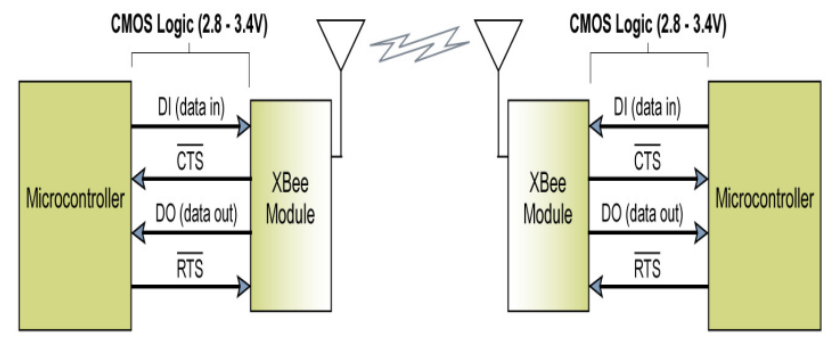

Fig3. Zigbee communication

\subsection{Micro controller family}

LPC2148 Microcontroller specifications:

1. It is a 32 bit, $64 \mathrm{MHz}$ processor with LQFP package.

2. It has $32 \mathrm{k}$ byte of SRAM, $512 \mathrm{k}$ bytes of FLASH MEMORY and 37 registers (16 general purpose registers)

3. It has two 32 bit timers

4. It has in built 10 bit 14 ADC channels and single 10 bit DAC. Two UART channels for serial communication

5. It has two general purpose ports $(\mathrm{p} 0, \mathrm{p} 1)$

The ARM7TDMI core is the industry's mostly used 32-bit embedded RISC microcontrollers. Optimized for cost and power-sensitive applications, the ARM7TDMI solution provides small size, high performance and low power consumption needed in embedded applications. The ARM7TDMI-S core is the synthesized version of the 
ARM7TDMI core, available in both VHDL and VERILOG. Optimized for flexibility and featuring an identical feature set to the hard macro cell, it improves time-to-market by reducing development time while allowing for increased design flexibility, and enabling $>>98 \%$ fault coverage.

The ARM7TDMI-S is a general purpose 32-bit microcontroller, which offers very low power consumption and high performance. The ARM architecture is based on Reduced Instruction Set Computer principles, and the related decode mechanism and instruction set is much simpler than those of micro programmed Complex Instruction Set Computers (CISC). This simplicity results in a high instruction throughput and impressive real-time interrupt response from a small and cost-effective processor core. All parts of the memory systems can operate continuously using pipeline techniques.

\subsection{GSM Technology}

A GSM modem is a wireless modem works with the GSM wireless network. A wireless modem behaves like a dual up modem (sends and receives through the fixed telephone network) while wireless modem sends and receives data through radio waves. This modem sends message to personnel automatically about field information This modem sends message to personnel automatically about field information. A GSM modem can be an external device or a PC Card. Typically, an external GSM modem is connected to a computer through a serial cable or a USB cable. A GSM modem in the form of a PC Card / PCMCIA Card is designed for use with personal laptops. It should be inserted into one of the PC Card / PCMCIA Card slots of a laptop. Like a GSM mobile phone, a GSM modem requires a SIM card from a wireless carrier to operate the GSM modem. As mentioned in SMS theory, personal computers use standard AT commands to control modems. Both dial-up modems and GSM modems support a common set of AT commands. We can use a GSM modem just like a dial-up modem.

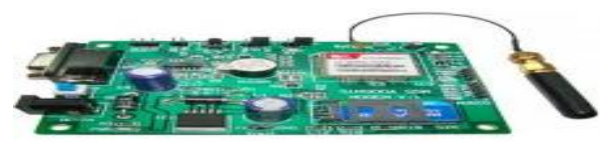

Fig4. GSM Module

SMS COMMANDS: Computer use AT commands to control modems. Both GSM modems and dual up modems supports a common set of AT commands. With AT commands administrator can do the things like

1. Reading, writing, deleting sms

2. Sending sms

3. Monitoring signal strength

4. Reading, writing and searching phone box entry.

5. AT plus CIMI

Note: It reads the IMSI

6. AT plus $C M G S="+9959372873$ "

Note: It sends a message in text mode

7. AT plus $C M G R=3$

Note: Reads it

\section{AT plus $C M G D=3$ \\ Note: Delete it}

\subsection{Humidity Sensor}

Amount of moisture level present in the atmosphere is called humidity. The amount of water vapour in the air can affect human comfort as well as many manufacturing processes in industries. So monitoring and controlling of humidity is important in many industrial and domestic applications. A humidity sensor designed for retrofit into a 1-Wire weather station is powered by and communicates over a single twisted pair. The HIH-3610 Series humidity sensor is designed specifically for high volume OEM (Original Equipment Manufacturer) users. Which is a direct input to a controller or other device is made possible by this sensor's linear voltage output. With a typical current draw of only $190 \mathrm{~mA}$, the HIH3610 Series is ideally suited for battery operated, low drain systems. Tight sensor interchange ability reduces or eliminates OEM production calibrating costs. Individual sensor calibration data is available. The HIH-3610 Series yields instrumentation-quality RH (Relative Humidity) sensing performance in a low cost, solder able Single In-line Package. The RH sensor is a laser thermo set polymer capacitive sensing element with on-chip integrated signal conditioning. The sensing element's multi-layer construction provides excellent resistance to application hazards such as wetting, dirt, dust, oils, and common environmental chemicals.

\subsection{Temperature Sensor}

The LM35 series are precision IC temperature sensors and output voltage of it's is linearly proportional to the Celsius temperature. So, it has an advantage over linear temperature sensors calibrated in ${ }^{\circ}$ Kelvin, as the user is not required to subtract a large constant voltage from its output to obtain convenient Centigrade scaling. The LM35 does not require any trimming or external calibration to provide typical accuracies of $\pm 1 / 4{ }^{\circ} \mathrm{C}$ at room temperature and $\pm 3 / 4{ }^{\circ} \mathrm{C}$ over a full -55 to $+150^{\circ} \mathrm{C}$ temperature range. Calibration and trimming at the wafer level assures low cost of device. The LM35's linear output, low output impedance and precise inherent calibration make interfacing to readout or control circuitry especially easy. LM35 uses single power supply, or with minus and plus supplies. As it draws only $60 \mu \mathrm{A}$ from its supply, it has low self-heating, less than $0.1^{\circ} \mathrm{C}$ in still air. The LM35 can operate over a $-55^{\circ}$ to $+150^{\circ} \mathrm{C}$ temperature range, while the $\mathrm{LM} 35 \mathrm{C}$ is rated for a $-40^{\circ}$ to $+110^{\circ} \mathrm{C}$ range. The LM35 series is available packaged in hermetic TO-46 transistor packages, while the LM35c, LM35d, and LM35a are also available in the plastic TO-92 transistor package.

\subsection{PH Sensor}

$\mathrm{pH}$ is an abbreviation of "pondus hydrogenii". $\mathrm{pH}$ is an important factor to be measured and controlled in the process world. Any solution has some $\mathrm{pH}$ value which indicates how acidic or basic (alkaline) it is and values of the hydrogen ion concentration is translated by $\mathrm{pH}$ term- which ranges between about 1 and $10 \times-14$ gram-equivalents per litre - into numbers between 0 and 14. On the $\mathrm{pH}$ scale an acidic solution has a low $\mathrm{pH}$ which corresponds to a large concentration of hydrogen ions, value such as 0,1 , or 2 . $(10 \times 0,10 \times-1$, or 10 $\mathrm{x}-2$ gram-equivalents per litre) while a very basic solution has a high $\mathrm{pH}$ value, such as $12 \_14$ which corresponds to a small number of hydrogen ions $(10 \mathrm{x}-12,10 \mathrm{x}-13$, or $10 \mathrm{x}-14$ gram-equivalents per litre). A solution such as water has a $\mathrm{pH}$ nearly equivalent to 7 .

A $\mathrm{pH}$ measurement loop is made up of 3 components, the $\mathrm{pH}$ sensor, which includes a reference electrode -ve terminal, 
measuring electrode +ve terminal and a temperature sensor; a pre-amplifier; and an analyzer or transmitter. $\mathrm{A} \mathrm{pH}$ measurement is essentially a battery where the +ve terminal is the measuring electrode and the -ve terminal is the reference electrode. The measuring electrode sensitive to the hydrogen ion, develops a potential (voltage) directly related to the ' $\mathrm{H}$ ' ion concentration of the solution. The reference electrode gives a stable potential against which the measuring electrode can be compared.

\subsection{Relay Unit}

The relay unit is connected to the motor which is deployed in the field and it is automatically monitored and controlled based on water level present in the field for that we deploy water level sensor in the field here ARM processor with embedded $\mathrm{C}$ programming helps to control the motor.

The coil of a relay passes a relatively large current, typically $30 \mathrm{~mA}$ for a 12 volts relay, but it can be as much as $100 \mathrm{~mA}$ for relays designed to operate from lower voltage levels. So a CB amplifier is used to achieve the current rating of the relay unit. ICs must be protected from the high voltage produced when a relay coil is switched off. The diagram shows how a signal diode (e.g. 1N4148) is connected 'backwards' across the relay coil to provide this protection.

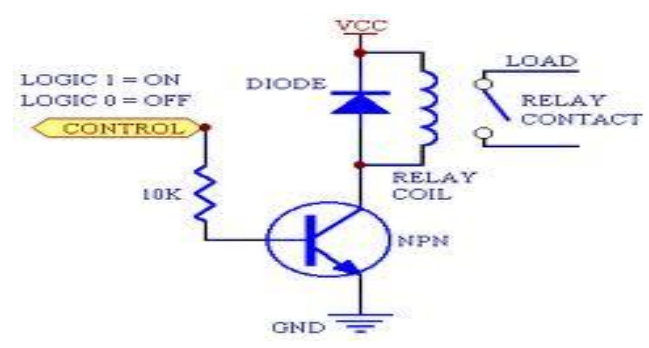

Fig5. Drive circuit and protection diodes for relays

\section{SOFTWARE TOOLS}

\subsection{OrCAD-Circuit Design}

The hardware schematic is designed using OrCAD circuit design tool.

\subsection{Keil IDE's}

The development of the source code needed for the design is done through keil IDE. This tool helps us not only to develop the source code but also compiles it and simulates it and convert the compiled Embedded $\mathrm{C}$ code to its equivalent hexadecimal code.

\subsection{Flash Programmer}

Flash programmer is used to fuse the built hexadecimal source code into the Microcontroller.

\subsection{Language}

Embedded C

\section{5 visual basic}

In monitoring section information will be displayed using Visual basic.

\section{FACTORS OF INTEREST AND SENSOR OUTPUT}

All the data from sensors will be sent wirelessly to central server then it will be analysed and monitored continuously. The system yields some essential factors for measurement:
$\square$ Temperature
Humidity
Water Level
Soil Moisture

\subsection{Parameter values in VB}

\section{Water Altitude 0.35 meters}

Humidity $\quad 3.61$

$$
\text { pH level } \quad 1.12
$$

Temperature $\quad 38$

\subsection{Resultant Graphs}

Humidity

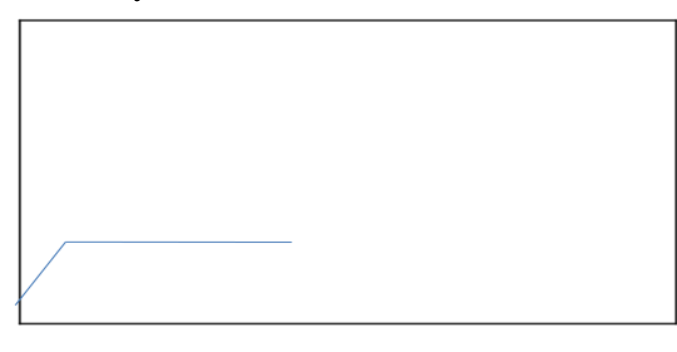

Time (in sec)

Fig6. Time vs. Humidity

Water level (in mm)

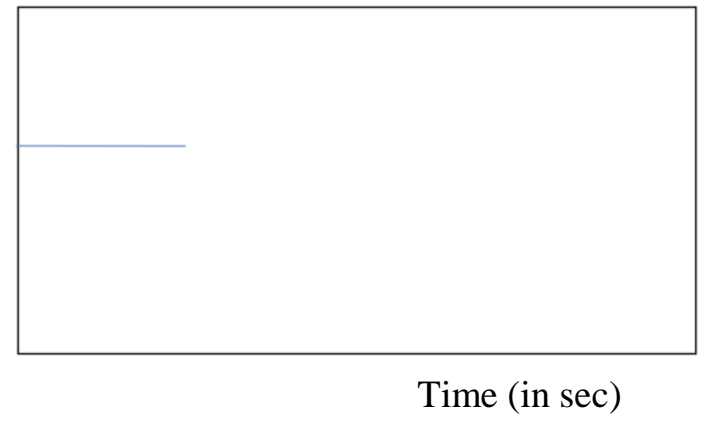

Fig7. Time vs. Water level

PH level 


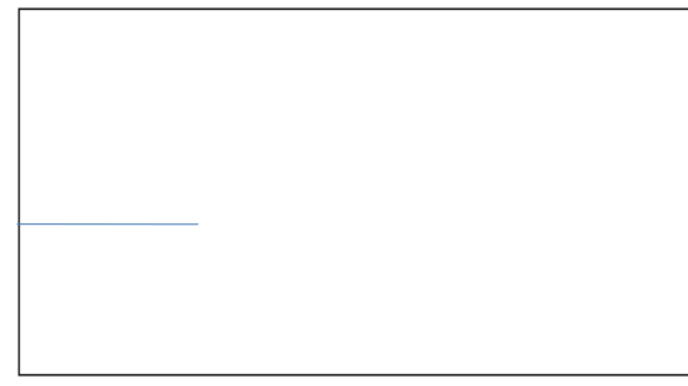

Time (in sec)

Fig8. Time vs. Ph level

Temperature (in degrees)

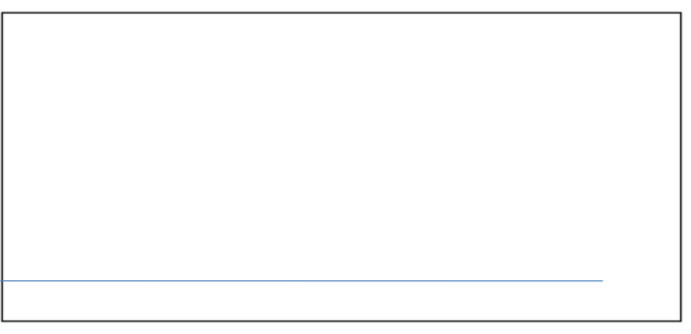

Time (in sec)

Fig9. Time vs. Temperature

\section{CONCLUSION}

Now a day's Indians are using manual techniques for Agricultural monitoring and controlling, due to that it will take time and it is not an effective System, for that we introduced new System.

In the new system a wireless communication and sensor technology is introduced, i.e. Humidity sensor, Temperature sensor, water level and $\mathrm{pH}$ sensor. By using these sensors above parameters can be estimated and information transferring through Zigbee wireless module to the crop field monitoring system. At field monitoring station if any sensor exceeds the set point value, it will send SMS by using GSM technology.

By using this new system administrator can monitor the field and control the field. This system minimizes water loggings in the field, avoids over fertilization, conserves water and minimizes power consumptions.

\section{REFERENCES}

[1] S. Blackmore. Precision farming: an introduction. Outlook on agriculture, 23(4):275-280, 1994.

[2] P. E. Drummond, C. D. Christy, and E. D. Lund, "Using an automated penetrometer and soil EC probe to characterize the rooting zone,"

[3] J. Song. Greenhouse monitoring and control system based on Zigbee wireless senor network. In 2010 International Conference on Electrical and Control Engineering, pages 2785-2788. IEEE.

[4] A ZigBee Based Embedded Control System, 2010 2nd International Conference on Signal Processing Systems (ICSPS).

[5] J. Hwang, C. Shin, and H. Yoe. A wireless sensor network-based ubiquitous paprika growth management system. Sensors, 10(12):11566-11589, Jan. 2010.

[6] C. Park, H. Cho, D. Park, Y. Lee, S. Cho, and J. Park. Aoa localization system design and implementation based on Zigbee for applying greenhouse. In 2010 5th International Conference on Embedded and Multimedia Computing. IEEE, Aug. 2010.

[7] ZHU Y Y.Multi-sensor intelligent video surveillance system based On DM642, [J].Chengdu, University of Electronic Science and Technology, 2008.

[8] WU Y L, PAN X B. Research and implementation of video processing system based on ARM and DSP Architecture [J].Electronic Design \& Application, 2009, 35(3):82-84. 